through on which all subsequent development was based. During the next hundred years, an increasing number of clinical reports of individual cases was published and Dr Leibowitz draws attention to a well documented account of rupture of the heart described by Harvey himself in 1649. But it was not until the eighteenth century that advances in clinical medicine and pathology began to parallel those which had taken place earlier in the basic sciences, and Heberden's classical description of angina pectoris, never bettered by any writer since, was published in 1769 . Within ten years disease of the coronary arteries was recognized as causing angina pectoris and by the end of the century the work of Parry, Jenner and others had clearly established the relationship between coronary artery disease, angina pectoris and sudden death. The nineteenth century saw the development of cellular and vascular pathology, but only in the twentieth century, with the advent of electrocardiography, was coronary heart disease in its manifold forms fully recognized and Dr Leibowitz unfolds a fascinating story of the developments which have taken place during the past fifty years. Investigation at present is directed specifically to the study of aetiology and prevention, and the book concludes with an up to date account of contemporary thought.

This book is a work of scholarship drawing on the widest possible range of sources and providing an excellent bibliography. Footnotes are well written and unobtrusive, yet provide useful small print information. There is an unexpected error in the identity of a Dr Anonymous who had left his body to $\mathrm{Dr}$ Heberden for dissection following his death from angina pectoris in $\mathbf{1 7 7 2}$. The autopsy failed to show any abnormality of the heart. Dr Leibowitz identified this public spirited patient as Dr Haygarth of Chester, a physician of outstanding repute in the field of infectious fevers. Haygarth, however, cannot have been Heberden's patient, for he lived on until 1827 when he died in Bath at the advanced age of 87 . Such minor points do not detract in any way from the excellence of this scholarly account of one of the major diseases of modern times.

\section{C. Booth}

\section{Catching the Light}

Invertebrate Photoreceptors: a Comparative Analysis. By Jerome J. Wolken. Pp. xi+179. (Academic: New York and London, May 1971.) $\$ 9.50 ; £ 4.45$.

IN spite of a generalized title, J. J. Wolken's little book is an illustrative rather than an exhaustive comparative study of invertebrate photoreceptors.
By way of introduction the book begins with a description of the electromagnetic spectrum and the physics of light, and this is combined with equally useful information concerning the classification and basic biochemistry of the photosensitive pigments found in plants and animals.

The comparative section begins with that group of animals which has been neglected perhaps the most by photobiologists-the Protozoa-illustrated in this case by the flagellate, Euglena gracilis. The fine structures of the flagellum and eyespot are described and experimental details of the animals' photobehaviour are integrated with microspectrophotometric studies of the various organs involved. This is followed by a consideration of the compound eye as found in many insects and Crustacea. Some nine genera of arthropods are described in detail, including the copepod Copilia, a species which has become ubiquitous in textbooks of late, presumably because of the perversely unusual visual apparatus it possesses. The invertebrate survey is concluded with a study of the structure of the photoreceptors found in the refracting eye of cephalopod molluscs, such as the squid. It was wise of the author to contrast all the invertebrate structures with the vertebrate retinal rod. The whole forms a good introduction to the study of photoreceptor structure and the subject is made all the more easy to grasp by virtue of the many fine micrographs which form the text figures.

The second part of the book is concerned with the vertebrate and invertebrate visual pigments and deals with techniques for their extraction and purification. The various absorption spectra as measured both in vitro and in vivo are then presented and discussed, as are similar results for the accessory and screening pigments found in association with the various photoreceptors.

An important generalization in the concluding section is that a functional difference exists between the "open" type of rhabdomere arrangement found in the dipterous insects and the "closed" or fused rhabdomeres of many insects and Crustacea (excepting, of course, Copilia which is of the open type). This difference is seen as the adaptation of the closed type to vision at low light intensities, which is fair enough except to say that many insects with closed type rhabdomeres, for example, the bees and wasps, the dragonflies and perhaps the locusts and grasshoppers, are as diurnal in habit as any of the open eyed Diptera. To correlate the structural findings with the electrophysiological evidence the author associates the open eye with the "fast" category of electroretinogram and the closed eye with the "slow" type. Once again the bees are usually considered as having the fast electrical responses, yet are shown to possess the closed rhabdomere arrangement, so that one feels the author should have presented a rather fuller argument for his interpretation of photoreceptor fine structure.

Omitting as it does almost all reference to the electrophysiological events associated with visual excitation, this book leaves the impression of a story unfinished, and this is one reason why Invertebrate Photoreceptors remains a good basis for the study of the subject but lacks the breadth to make it a thoroughgoing comparative account.

JOHN PATTERSON

\section{Snakes of Two Regions}

A Field Guide to the Snakes of Southern Africa. By V. F. M. FitzSimons. Pp. $221+12$ plates. (Collins : London, January 1971.) £2.10.

The Snakes of Europe. By J. W. Steward. Pp. $238+28$ photographs. (David and Charles: Newton Abbot, March 1971.) £2.75.

EUROPE has thirty-three species of snakes, only five of them confined to the area. There was a general work on European reptiles nearly a hundred years ago and a book devoted to the snakes in particular nearly sixty years ago. Mr Steward brings us up to date on the basis of years of field and vivarium experience and much travel. Southern Africa has three and a half times the number of species, about fortytwo of them endemic, and may well have undiscovered forms. Dr FitzSimons's handbook is based on his own pioneering studies in the museum and the field.

Both works recognize a considerable number of subspecies. Although both authors sometimes pause to express doubts about the status of some taxa neither of them discusses the nature of subspecies and makes it clear that they are subjective categories; the existence of zones of intermediacy is barely hinted. Surely the non-specialist would have been better served by an informal survey of the variant regional populations, some of which have been recognized as subspecies. I make this comment partly because I believe that, in many cases, when a larger number of characters is studied, many of them will be found to vary independently and many of the subspecies boundaries be seen to be arbitrary.

Both authors give aids to identification but neither takes the reader gently by the hand through the problems nor mentions museums as a source of information. FitzSimons groups most, but not all, of the snakes by predominant colour and pattern. If one's snake be mostly brown, however, there are fiftynine to choose from; this can be narrowed further if it be banded, 